\title{
The Need for Differential Diagnosis in Cleft Palate Treatment Planning
}

\section{Samuel Berkowitz*}

DDS, MS, FICD, Adjunct Professor - Orthodontics, Illinois School of Dentistry, Chicago, Illinois, USA

Although the treatment of children with cleft lip/palate and other types of craniofacial anomalies in the United States has improved dramatically, many children with cleft lip and palate still receive care that is substantially inferior to what can or should be provided. Inadequate care results from diagnostic errors, failure to recognize and treat the full spectrum of health problems associated with complete unilateral cleft lip and palate (CUCLP) and complete bilateral cleft lip and palate (CBCLP), unnecessary and poorly timed treatment and inappropriate or poorly performed procedures. The answer to these problems lies in not obtaining longitudinal serial records of dental casts, lateral cephs, photographs and the use of the nasopharyngoscope.

Those clinicians $[1,2]$ who have reviewed the longitudinal results of presurgical orthopedics with gingivoperiosteoplasty and early palate closure [3], will take a contrary view and will argue against the need to do early surgical palatal closure and enhance the need for the attainment of improved palatal growth focusing on the cleft width relative to the amount of available mucoperiosteal tissue and the facial growth pattern. To do so will lead to good facial/palatal growth and functional dental occlusion as well as acceptable speech and more successfully treated cleft palate cases.

Today, most surgeons [4,5] main thrust is to achieve early lip/ nose aesthetics even when realizing it occurs at the expense of normal midfacial development. It goes without saying that professionals entrusted with the provision of health care have an obligation to review the success of their practices and where shortcomings are revealed, to take remedial action. Cycles of outcome audit are more easily established when the intervention is common and the consequences are clear-cut and quickly observable. Cleft audit, therefore, involves a considerable challenge because of the lengthy follow up required, the complexity of inter-center collaboration and record review [6-8]. It still offers significant advantages by providing insights into the processes and outcomes on treatment of comparable services.

\section{Condemnation of Presurgical Orthopedics and Periosteoplasty}

One of the most widely debated areas in the treatment of cleft lip and palate still under scrutiny involves the use of presurgical orthopedics and periosteoplasty such as presurgical orthopedics with periosteoplasty and lip adhesion (POPLA) designed by Ralph Latham and D. Ralph Millard Jr. [9-11] and now by Cutting and Grayson [5]. They modified the mechanics and now call it NAM + GPP (nasoalveolar molding + gingivoperiosteoplasty. They contend that it is an extremely beneficial concept that is superior for producing more aesthetically appealing lip/nose surgery, while still allowing for good midfacial growth. POPLA and GPPP +NAM proponents believe the use of presurgical orthopedics (PSO) procedures in complete bilateral cleft lip and palate (CBCLP) patients, which forcefully retracts the protruding premaxilla, favors the attainment of improved facial aesthetics and palatal arch alignment soon after birth. They speculate that the early aesthetic benefits will remain as the face grows and develops.

In both procedures, presurgical orthopedics is followed by periosteoplasty in the hope that the bone-bridging created will avert the need for secondary alveolar bone grafts at a later date. The same reasons are given for performing early palatal manipulation with periosteoplasty in complete unilateral cleft lip and palate (CUCLP).
Hsieh et al. [12] however, reported in a serial cephalometric study that NAM + GPP caused severe midfacial recessiveness in POPLA, CBCLP and some CUCLP cases.

It is not a simple or a lightly assumed task to offer a brief, challenging the rationale for presurgical orthopedics and periosteoplasty for infants with complete unilateral or complete bilateral cleft lip and palate. Advocates of the POPLA and NAM + GPP concepts are few and for them the proposed goal of making things right and whole as early as possible seems sensible and has great emotional appeal.

\section{The Need for Additional Quantitative Data}

The literature on early priorities has never lead to a consensus of treatment due to early specialist's channeled thinking based on a dearth of properly designed multicenter, multidisciplinary clinical research studies. Each specialist has failed to consider the many interdependent anatomical and functional aspects of the face and nasopharynx that control facial/palatal growth, speech and dental occlusion. Multispecialty studies require extensive testing and longitudinal objective records extending from birth through adolescence that focus on evidence-based differential diagnosis for treatment planning [13].

The cleft palate classification system lumps all cleft cases according to the physical type and disregards variations in the size of the cleft defect in the surgical time decision. Because each cleft defect size may differ in the amount of osteogenic deficiency, each patient must be treated differently according to his or her physical assets and deficits and not on age alone $[1,13]$. There is a lack of consensus as to the importance of the relative size of the cleft space to surrounding mucuperiosteal tissue medial to the alveolar ridges and what and when surgical procedures should be performed to achieve all treatment goals of good facial aesthetics, speech and dental occlusion without priority being given to one goal over another. Without such studies we will remain caught in an unchanging time/treatment warp with a fixed mindset suggesting that compromises must exist and that patients may have poor speech and poor dental occlusion as long as the nasal and lip aesthetics are the only goals to be achieved. The speech language pathologists Chapman [14], on the other hand, usually vote for good speech above everything else.

Many longitudinal studies have shown that the best time to perform palatal surgical closure of CUCLP and CBCLP cases is between 18-24 months in most cases, and sometimes even later depending on the ratio of the cleft space to the size of palatal mucoperiosteum medial to the alveolar ridge.

*Corresponding author: Samuel Berkowitz, DDS, MS, FICD, Adjunct Professo - Orthodontics, Illinois School of Dentistry, Chicago, Illinois, USA, Tel: +305-6618815; E-mail: sberk3140@aol.com

Received February 10, 2014; Accepted February 27, 2014; Published March 01, 2014

Citation: Berkowitz S (2014) The Need for Differential Diagnosis in Cleft Palate Treatment Planning. Dentistry 4: 218. doi:10.4172/2161-1122.1000218

Copyright: (c) 2014 Berkowitz S. This is an open-access article distributed under the terms of the Creative Commons Attribution License, which permits unrestricted use, distribution, and reproduction in any medium, provided the original author and source are credited. 


\section{References}

1. Berkowitz S, Mejia M, Bystrik A (2004) A Comparison of the Effects of the Latham-Millard Procedure with Those of a Conservative Treatment Approach for Dental Occlusion and Facial Aesthetics in Unilateral and Bilateral Complete Cleft Lip and Palate: Part I. Dental Occlusion. Plast Reconstr Surg 113: 1-18.

2. Berkowitz S, Duncan R, Evans C, Friede H, Kuijpers-Jagtman AM, et al. (2005) Timing of cleft palate closure should be based on the ratio of the area of the cleft to that of the palatal segments and not on age alone. Plast Reconstr Surg 115: 1483-1499.

3. Berkowitz S (2013) Cleft Lip and Palate: Diagnosis and Management. (3rdedn), Springer-Verlag, Heidelberg, New York.

4. Cosman B, Falk AS (1980) Delayed hard palate repair and speech deficiencies: a cautionary report. Cleft Pal J 17: 27-33.

5. Cutting C, Grayson BH (2000) Presurgical nasoalveolar orthopedic molding in primary correction of the nose, lip and alveolus of infants born with unilateral and bilateral clefts. Cleft Pal J 37: 528-532.

6. Pruzansky S (1953) Description, classification and analysis of unoperated clefts of the lip and palate. Am J Orthod 39-59.
7. Pruzansky S (1971) The growth of the premaxillary vomerine complex in complete bilateral cleft lip and palate. Tandlaegebladet 75: 1157-1169.

8. Ross RB (1987) Treatment variables affecting facial growth in complete unilateral cleft lip and palate. Cleft Pal J 24: 5-77.

9. Latham RA (1980) Orthopedic advancement of the cleft maxillary segment: a preliminary report. Cleft Pal J 17: 227-233.

10. Millard DR Jr (1986) Cleft Craft: Part III - The Evolution of its Surgery: Alveolar and Palatal Deformity. Little Brown, Boston.

11. Millard DR Jr, Latham RA (1990) Improved surgical and dental treatment of clefts. Plast Reconst Surg 86: 856-871.

12. Hsieh CH, Ko EW, Chen PK, Huang CS (2010) The Effects of Gingivoperiosteoplasty on Facial growth in Patients with Complete Unilateral Cleft Lip and Palate. Cleft Palate Craniofac J 47: 439-446.

13. Berkowitz S (2006) Timing of cleft palate closure should be based on the ratio of the area of the cleft to that of the palatal segments and not on age along. Springer-Verlag Heidelberg, New York.

14. Chapman KL (1993) Phonologic processes in children with cleft palate. Cleft Palate Craniofac J 30: 64-72. 